Document downloaded from:

http://hdl.handle.net/10251/60021

This paper must be cited as:

Molina, S.; Salvador Rubio, FJ.; Carreres Talens, M.; Jaramillo, D. (2014). A computational investigation on the influence of the use of elliptical orifices on the inner nozzle flow and cavitation development in diesel injector nozzles. Energy Conversion and Management. 79:114-127. doi:10.1016/j.enconman.2013.12.015.

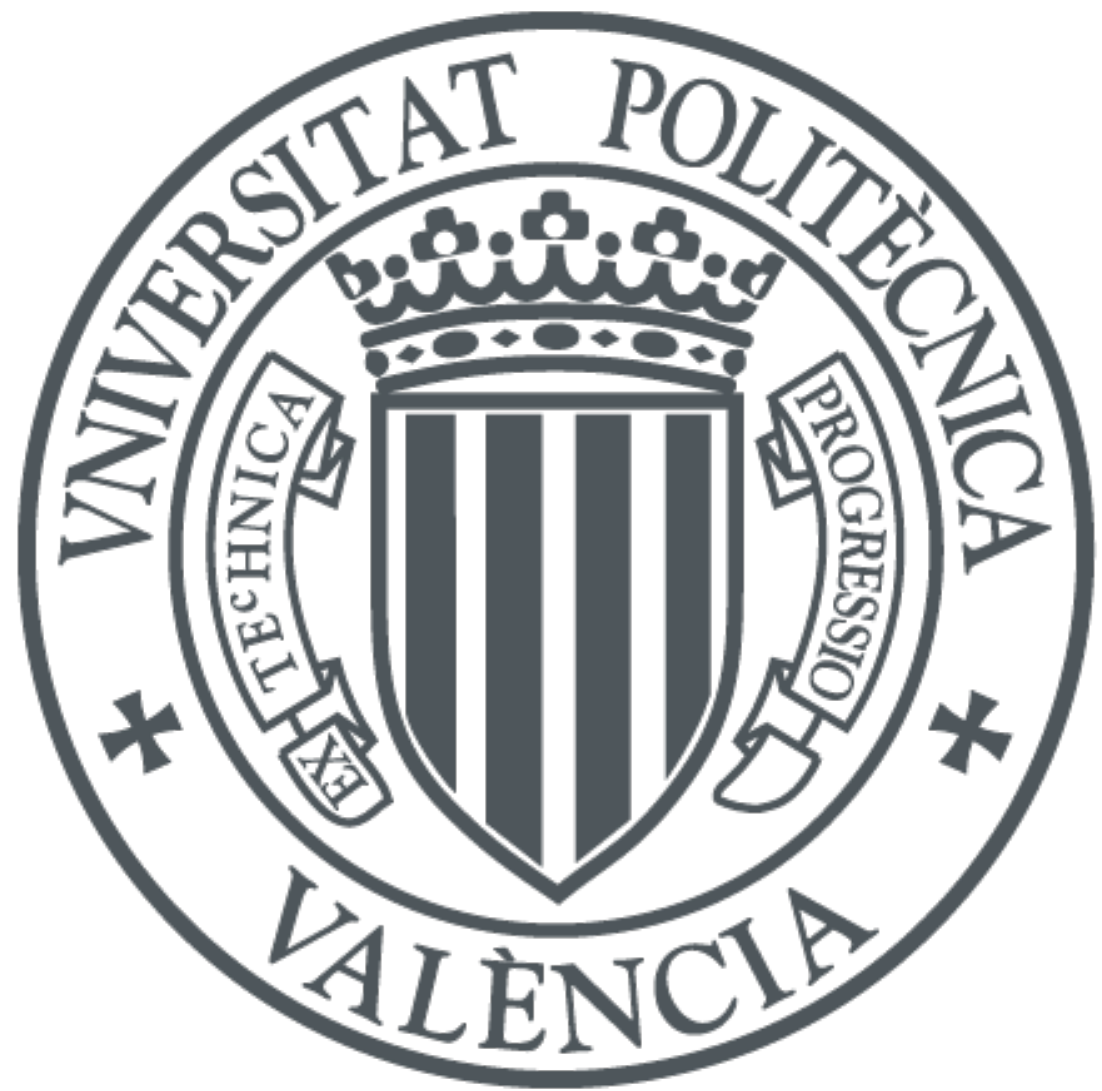

The final publication is available at

http://dx.doi.org/10.1016/j.enconman.2013.12.015

Copyright Elsevier

Additional Information 


\section{A COMPUTATIONAL INVESTIGATION ON THE INFLUENCE OF THE USE OF ELLIPTICAL ORIFICES ON THE INNER NOZZLE FLOW AND CAVITATION DEVELOPMENT IN DIESEL INJECTOR NOZZLES.}

Molina, S., Salvador, FJ (*), Carreres, M., Jaramillo, D.

CMT-Motores Térmicos, Universitat Politècnica de València

Camino de Vera s/n, E-46022 Spain.

(*) Corresponding author:

Dr. F. Javier Salvador, fsalvado@mot.upv.es

CMT-Motores Térmicos, Universitat Politècnica de València

Camino de Vera s/n, E-46022 Spain.

Telephone: +34-963879659

FAX: +34-963877659 


\section{ABSTRACT}

In this paper a computational study was carried out in order to investigate the influence of the use of elliptical orifices on the inner nozzle flow and cavitation development. With this aim, a large number of injection conditions have been simulated and analysed for 5 different nozzles: four nozzles with different elliptical orifices and one standard nozzle with circular orifices. The four elliptical nozzles differ from each other in the orientation of the major axis (vertical or horizontal) and in the eccentricity value, but keeping the same outlet section in all cases. The comparison has been made in terms of mass flow, momentum flux and other important non-dimensional parameters which help to describe the behaviour of the inner nozzle flow: discharge coefficient $\left(C_{d}\right)$, area coefficient $\left(\mathrm{C}_{\mathrm{a}}\right)$ and velocity coefficient $\left(\mathrm{C}_{\mathrm{v}}\right)$. The simulations have been done with a code able to simulate the flow under either cavitating or non-cavitating conditions. This code has been previously validated using experimental measurements over the standard nozzle with circular orifices. The main results of the investigation have shown how the different geometries modify the critical cavitation conditions as well as the discharge coefficient and the effective velocity. In particular, elliptical geometries with vertically oriented major axis are less prone to cavitate and have a lower discharge coefficient, whereas elliptical geometries with horizontally oriented major axis are more prone to cavitate and show a higher discharge coefficient.

\section{KEYWORDS}

diesel, injection, nozzle, elliptical nozzle, cavitation.

\section{LIST OF NOTATION}

A area

$A_{\text {eff }}$ outlet effective area 
$A_{o} \quad$ outlet area

a major axis of the ellipse

b minor axis of the ellipse

$C_{a}$ area coefficient, $C_{a}=\frac{A_{e f f}}{A_{o}}$

$C_{d}$ discharge coefficient, $C_{d}=\frac{m_{f}}{\rho_{l} A_{o} u_{t h}}=\frac{m_{f}}{A_{o} \sqrt{2 \rho_{l} \Delta P}}=C_{v} C_{a}$

$C_{v} \quad$ velocity coefficient, $C_{v}=\frac{u_{e f f}}{u_{t h}}$

c speed of sound

$D_{\text {eff }}$ effective diameter

$D_{H}$ Hydraulic diameter

$D_{i} \quad$ diameter at the orifice inlet

$D_{o}$ diameter at the orifice outlet

e eccentricity

K cavitation number

$k \quad$ turbulent kinetic energy

$k$-factor orifice conicity factor

L orifice length

$\dot{M}_{f} \quad$ momentum flux 
$\dot{m}_{f}$ mass flow

$P \quad$ orifice wetted perimeter

p pressure

$P_{b} \quad$ discharge back pressure

$P_{i} \quad$ injection pressure

$p_{\text {sat }}$ vaporisation pressure

$r \quad$ rounding radius at the inlet orifice

$t$ time

$u \quad$ velocity

$\bar{u} \quad$ averaged velocity

$u_{e f f}$ effective velocity, $u_{e f f}=\frac{\dot{M}_{f}}{\dot{m}_{f}}$

$u_{t h}$ theoretical velocity, $u_{t h}=\sqrt{\frac{2\left(P_{i}-P_{b}\right)}{\rho}}$

\section{GREEK SYMBOLS:}

$\Delta P$ pressure drop, $\Delta P=P_{i}-P_{b}$

$\Psi \quad$ fluid compressibility

$\Psi_{l} \quad$ liquid compressibility 
$\Psi_{v} \quad$ vapour compressibility

$\gamma \quad$ vapour mass fraction

$\mu \quad$ fluid viscosity

$\mu_{l} \quad$ liquid viscosity

$\mu_{v} \quad$ vapour viscosity

$\rho \quad$ fluid density

$\rho_{l} \quad$ liquid density

$\rho_{\text {lsat }}$ liquid density at saturation

$\rho_{l}^{o} \quad$ liquid density at a given temperature condition

$\rho_{\text {vsat }}$ vapour density at saturation

$\rho_{v} \quad$ vapour density

\section{INTRODUCTION}

With further demands in the future to reduce the emission levels for diesel engines, after treatment devices like particulate traps and selective catalytic reduction seem inevitable. Even though those devices will be necessary, it is important to keep the raw emissions low in order to minimize the cost and the complexity of the devices. The main emissions of diesel engines are particulates and nitrogen oxides. The characteristics of the diesel engine tend to trade off these emissions, so that one emission is increased when the other one is reduced. Finding a technical solution that reduces both kind of emissions (or at least reduces one while the second remains unchanged over most of 
the engine operating range) is of great interest. One of the most important processes controlling the combustion efficiency and therefore the emissions formation is the air-fuel mixing process. It is well known that the time for the combustion kinetics is fast compared to the time of mixing ([1]). The mixing process is therefore controlling the combustion. Once the fuel is injected into the combustion chamber, the fuel mixes with air and vaporizes. The air-fuel mixing process depends on spray characteristics, which in turn depend on the injection pressure and the dimensions and geometry of the nozzle orifice among other factors ([2][3][4][5][6]).

Some investigations in the literature have shown that air entrainment of jets injected into gas is considerably increased if elliptic orifices are used instead of circular orifices ([7][8]). In other studies where liquid fuel was injected into gas trough elliptical nozzles researchers have shown that the spray cone angle in the minor axis plane is higher than the spray cone angle in the major axis plane, indicating that air entrainment is enhanced ([9]). The same behaviour has been shown in more recent research; for example in Lee et al. [10] who compared an elliptical geometry nozzle hole with a conventional cylindrical one. From the main results of their study, it was shown that the spray tip penetration became shorter and the spray cone angle became wider with the elliptical geometry due to the fast break up of the fuel liquid column. Hong et al. [11] also studied the effect of elliptical nozzles on the spray characteristics and they observed a larger spray angle in the elliptical nozzle than in the circular nozzles.

Since improved air entrainment could have positive effects on the emissions of NOx and particulates, Matsson et al. [12] performed an investigation on the influence of non-circular orifice geometries. The tests were made using a passenger car diesel engine and a single cylinder engine with optical access. The results showed a reduction in NOx for the nozzle with elliptical orifices in comparison with the reference circular nozzle. Nevertheless, the use of elliptical nozzles did not affect on soot particulates. 
Although there are a lot of referenced works in the literature, most of them, with exception of Hong et al. [11], aim at analysing the influence of the use of elliptical nozzles on the spray and combustion process, but treating the nozzle itself as a black box, thus without investigating which are the changes in the flow pattern inside the nozzle that promote the improvement of the air-fuel mixing process through the increment of the spray cone angle. Only Hong et al. [11] have tried to link the internal nozzle flow characteristics in terms of cavitation appearance and intensity to the shape of the spray when comparing circular and elliptical nozzle holes. With this aim, they used non-conventional transparent acrylic symmetric models for visualization purposes. The crosssectional areas of circular and elliptical nozzles were the same and based on a 3mm diameter circular nozzle, therefore quite far from being representative values for standard nozzle hole diameters in Diesel injectors (0.1-0.2 mm). The authors reported a lower discharge coefficient for the elliptical nozzles and a lower discharge coefficient than that of the circular nozzle at the same Reynolds number.

The aim of the present paper has been to evaluate the potential of using non-conventional elliptical nozzle holes by means of CFD (computational fluid dynamics) calculations, comparing the results of four different elliptical nozzles with a base standard circular nozzle hole. The comparison has been made in terms of mass flow, momentum flux and other important non-dimensional parameters which are important features describing the behaviour of the inner nozzle flow: discharge coefficient $\left(\mathrm{C}_{\mathrm{d}}\right)$, area coefficient $\left(\mathrm{C}_{\mathrm{a}}\right)$ and velocity coefficient $\left(\mathrm{C}_{\mathrm{v}}\right)$. Furthermore, the fact that nonconvergent holes are used makes the nozzles prone to cavitate ([13]).Thus, critical conditions for cavitation inception, its development and morphology have also been studied and analysed.

The final objective is to identify a connection between the different expected flow pattern in elliptical nozzles and the different spray cone angle behaviour confirmed in the experiments referenced above. If we consider two different nozzles, the former with elliptical orifices and the 
latter with circular orifices, since the elliptical orifice has a bigger perimeter for the same crosssectional area, atomization due to air-fuel interaction is supposed to be enhanced, which is in agreement with the experimental results. Nevertheless, there should also be other reasons as far as flow characteristics are concerned: differences in flow regime, cavitation inception and development, effective injection velocity, etc. The present paper will be focused on this kind of differences.

For that purpose, a Homogeneous Equilibrium Model able to model the cavitation phenomenon was used. The code is implemented in the version 1.5 of OpenFOAM ${ }^{\mathrm{R}}$ [14]. This code has been extensively validated with mass flow measurements, momentum flux measurements and injection velocity at the nozzle exit. The validation was done over one of the nozzles used in the present investigation: the standard nozzle with circular orifices. The results obtained from simulations and their comparison with the experimental data showed that the model is able to predict the behaviour of the fluid in both cavitating and non-cavitating conditions with high level of confidence.

As far as the structure of the paper is concerned, it has been divided in 6 sections. First of all, in section 2, a description of the CFD approach is made, where the equations governing the model and how they are solved are explained in detail. Following, the geometry characteristics of the five nozzles, the injection conditions (injection pressure and backpressure) and fluid properties used for the simulations are described in section 3 where a validation of the cavitation model is also shown. In section 4, results of mass flow, momentum flux and flow coefficients are presented and analysed for all the nozzles. A special attention is paid to cavitation inception conditions and morphology in the different nozzles tested. The influence of cavitation on the flow parameters is widely analysed and discussed in this section. Due to the high volume of results and comparisons, a synthesis is presented in section 5, mainly focusing on the mixing process. Finally, the main conclusions of the investigation are drawn in section 6. 


\section{DESCRIPTION OF THE CFD APPROACH}

\subsection{Cavitation modelling.}

As explained in [15] three approaches are mainly considered for modelling cavitation: Interface tracking methods, two-fluid flow models and continuum nozzle flow models or homogeneous equilibrium models (HEM models). In the case we are dealing with, the use of a HEM with a barotropic equation of state seems to be the most suitable method due to the high pressures and velocities that occur within diesel injectors. The code assumes that both liquid and vapour phases are completely mixed in each cell and the compressibility of both phases is taken into account.

In the code implemented in OpenFOAM, a barotropic equation that relates pressure and density derivatives is used as a closure of the Navier-Stokes equations, where $\Psi$ is the fluid compressibility, defined as the inverse of the squared speed of sound:

$$
\begin{aligned}
& \left(\frac{\partial \rho(t, p(\vec{x}, t))}{\partial p}\right)_{t}=\Psi \\
& \Psi=\frac{1}{c^{2}}
\end{aligned}
$$

The amount of vapour in the fluid is calculated with the void fraction $\gamma$, where the extreme conditions $\gamma=0$ and $\gamma=1$ indicate that only one phase is presented in the fluid: $\gamma=0$ for noncavitating conditions (only liquid phase); $\gamma=1$ for fully cavitating conditions (only vapour phase).

$$
\gamma=\max \left(\min \left(\frac{\rho-\rho_{l, s a t}}{\rho_{v, s a t}-\rho_{l, s a t}}, 1\right), 0\right)
$$

The compressibility of the mixture is calculated from $\Psi_{v}$ and $\Psi_{v}$ (vapour and liquid compressibility, respectively) using a linear model. Although more accurate and complicated models can be found in 
the literature (Chung [16] and Wallis [17]), the linear model has been chosen due to its convergence and stability.

$$
\Psi=\gamma \Psi_{v}+(1-\gamma) \Psi_{l}
$$

In the case where there is only vapour or liquid, the following linear equation of state can be derived from equation (1) if the speed of sound is considered constant:

$$
\begin{aligned}
& \rho_{v}=\Psi_{v} p \\
& \rho_{l}=\rho_{l}^{0}+\Psi_{l} p
\end{aligned}
$$

The linear model has also been used to calculate the density and the viscosity of the mixture.

$$
\begin{aligned}
& \rho=(1-\gamma) \rho_{l}^{0}+\Psi \cdot p \\
& \mu=\gamma \mu_{v}+(1-\gamma) \mu_{l}
\end{aligned}
$$

The iteration process to numerically solve the fluid behaviour starts with the continuity equation (7) to get a provisional density.

$$
\frac{\partial \rho}{\partial t}+\nabla \cdot(\rho \vec{u})=0
$$

According to previous studies performed by the authors [15], the divergence term $\nabla \cdot(\rho \vec{u})$ is discretized in the space by using a Gauss upwind scheme to improve the stability, whereas an implicit discretisation in time is used for the density in the divergence term. With respect to the partial derivative over time, an Euler scheme is used for time discretisation. When the provisional density is computed, preliminary values for $\gamma$ and $\Psi$ are determined using equations (3) and (4). The next step is the calculation of a predictor for the velocity from the momentum conservation 
equation (equation (8)). The same procedure as before is followed: an Euler scheme for the partial derivatives over time and a Gauss upwind scheme for the divergence terms.

$$
\frac{\partial(\rho \vec{u})}{\partial t}+\nabla \cdot(\rho \vec{u} \cdot \vec{u})=-\nabla p+\nabla \cdot\left(\mu\left(\nabla \vec{u}+\nabla \vec{u}^{T}\right)\right)
$$

Then the continuity equation (equation (7)) is modified with the equation of state (equation (5)) and the following equation is solved by an iterative PISO algorithm:

$$
\frac{\partial(\Psi p)}{\partial t}-\left(\rho_{l}^{0}+\left(\Psi_{l}-\Psi_{v}\right) p_{s a t}\right) \frac{\partial}{\partial t}-p_{s a t} \frac{\partial \Psi}{\partial t}+\nabla \cdot(\rho \vec{u})=0
$$

When the continuity convergence has been reached, the variables $\rho, \gamma$ and $\Psi$ are updated using the equations (5), (4) and (3), and the PISO algorithm is started again until convergence.

\subsection{Turbulence modelling}

The turbulence is modelled using a RANS (Reynolds-averaged Navier-Stokes) method. A RNG k- $\varepsilon$ model [18] is used for the present work.

The selection of this model is based on three different reasons:

From the theoretical point of view:

- This model is supposed to provide improved predictions compared to the standard k- $\varepsilon$ in the case of simulations of flows with high streamline curvature and zones with flow detachment. These are both typical characteristics of multi-hole nozzles.

- The way it models the effective viscosity makes it possible to better handle low-Reynoldsnumber flows and near-wall flows. In the case of Diesel nozzles wall effects are extremely important because of the small dimensions of the orifices diameter. As far as the Reynolds 
number is concerned, it depends on the injection conditions and, as it can be seen in section 4.3, it can vary from relatively low values, about 5000 (at $30 \mathrm{MPa}$ of injection pressure), to high values about 25000 (at 160 MPa of injection pressure). Therefore, since a wide range of variation of Reynolds number is expected, RNG k- $\varepsilon$ is expected to be a more suitable turbulence model than standard k- $\varepsilon$ due to its better adaptability to Reynolds number variations.

- From a practical point of view, when comparing computational results with experiments for validation purposes the RNG k- $\varepsilon$ model provides the results which are closer to the experimental ones.

\section{NUMERICAL SIMULATIONS DESCRIPTION}

\subsection{Nozzles geometry and calculation set-up}

The computational study was performed over five six-hole microsac nozzles: four of them with a non-conventional (elliptical) orifice shape and one with a standard circular orifice shape. In Figure 1 , the different topologies of tested nozzles are depicted. Because of nozzle geometry and in order to speed up the calculations, the domain has been reduced to (1/6) i.e. $60^{\circ}$ of the total nozzle. The geometries represent full needle lift conditions (i.e. $250 \mu \mathrm{m}$ ). As it can be seen from that Figure, apart from the standard nozzle (so-called circular in the Figure), there are four elliptical nozzles. Nozzles so-called Elip-H1 and Elip-H2 have the major axis of the ellipse horizontally oriented and differ from each other in the eccentricity value. Nozzles so-called Elip-V1 and Elip-V2 have the major axis vertically oriented and also differ in their eccentricity value.

Table 1 describes the geometrical parameters of all nozzles according to the nomenclature used in 
Figure 2. In the Table, the eccentricity, $e$, evaluates the difference between the elliptical cross section and a circular section. It is defined as:

$e=\sqrt{1-(b / a)^{2}}$

where $a$ and $b$ are the major and minor axis of the ellipse, respectively. For a circular section, the eccentricity is equal to zero. For an elliptical section, the closer the eccentricity is to 1 , the higher the level of deformation ( $a$ and $b$ take very different values). In our case, Elip-H1 and Elip-V1 nozzles have the same eccentricity value $(0.734)$ with a relative low level of deformation with regards to the circular one, whereas Elip-H2 and Elip-V2 nozzles show a higher degree of eccentricity (0.866) and therefore higher level of deformation when compared to the circular one. To isolate the influence of the eccentricity and orifice shape, the cross sectional area is the same in all cases and equivalent to the area of a circular section of diameter equal to $170 \mu \mathrm{m}$.

The perimeter is calculated using the equation (11).

$P=4 \cdot a \cdot E(e)$

Where $E(e)$ is a function that depends on eccentricity and is defined as:

$E(e)=\int_{0}^{\pi / 2} \sqrt{1-e^{2} \sin ^{2} \theta} d \theta$

This parameter is useful when plotting the flow parameters as a function of the Reynolds number, which in turns depends on the wet perimeter.

As far as the other geometrical parameters are concerned, such as the rounding radius $(r)$, orifice length $(L)$ and nozzle angle $(\theta)$, the same and quite standard values have been chosen for all cases ([19][20]).

For the investigation, the geometries have been discretised in hexahedral cells keeping a quite 
uniform structured grid with a small transition zone just before the orifice inlet. A detail of the mesh is given in Figure 4. Sensitivity studies of the mesh performed in previous studies ([20]) made it possible to choose the most appropriate mesh refinements for the RANS calculations. As a result of those studies, it was established that the cell size in the hole should vary from around $7 \mu \mathrm{m}$ in the orifice core to a minimum value of $1.15 \mu \mathrm{m}$ in the near-wall region. For the rest of the cells in the nozzle, the cell size is fixed to $22.5 \mu \mathrm{m}$. With this cell size, the final mesh has around 240,000 cells.

As an indicator of the mesh quality, the mean and maximum values for the dimensionless distance, $\mathrm{y}^{+}$, were evaluated in the whole domain and for every nozzle. A distinction has been made between the orifice (where cavitation appears and most severe gradients of pressure and velocity are expected) and the rest of the geometry because of the different refinement requirements. The maximum and mean values of $y^{+}$in the orifice's wall are 5 and 1 , respectively, whereas the same values for the rest of the walls are 15 and 5 as can be seen in Figure 5 where the mean $y^{+}$values is depicted for all the nozzles. This result belongs to an operating point of $160 \mathrm{MPa}$ of injection pressure and 3MPa of discharge pressure, and, therefore, it has been calculated under the most severe pressure drop conditions among the total simulated points.

The grid infrastructure of the Spanish National Grid Initiative (es-NGI) [21] was used to perform some of the computation of the simulations. This grid infrastructure is part of the European Grid Initiative [22] that provides a stable Grid infrastructure for e-Science at European level. The es-NGI is composed of 15 sites with more than 12,000 CPUs and 300 TB of storage space. The calculations for this problem used above 42,000 CPU hours. Furthermore, the supercomputer Tirant at the University of Valencia (Spain) [23] was also used to perform a vast number of simulations(in particular those simulating high backpressures). In this case the calculations used above 15,000 CPU hours. More details about the grid application are given in [21][22]. 


\subsection{Boundary conditions and fluid properties}

The importance of choosing an appropriate set-up for the boundary conditions in order to ensure the convergence and the accuracy of the simulations is well-known. In our case, as depicted in Figure 3, a fixed pressure condition has been used at the inlet, where the injection pressure is set, whereas a mean pressure condition has been established for the outlet (backpressure). The mean pressure condition keeps the mean desirable value, allowing zones with very low pressures as a result of the presence of vapour in the flow. This boundary avoids the imposition of a rigorous pressure outlet that could affect the vapour structures developed as a consequence of the cavitation phenomenon. A non-slip condition for the velocity has been used at the walls. Finally, symmetry conditions have been employed at the symmetry surfaces.

In order to extensively characterize and compare all nozzles in both cavitating and non-cavitating conditions, two representative injection pressures of real engine running conditions have been considered: $160 \mathrm{MPa}$ and $30 \mathrm{MPa}$. As far as the backpressure is concerned, 7 different values have been simulated for the case of $30 \mathrm{MPa}$ and 17 different values for the case of $160 \mathrm{MPa}$. All the pressure conditions simulated can be seen in Table 2. The aforementioned simulations pursued a double goal: on one hand, to characterize and compare all nozzles in a wide range of Reynolds number and so, from a smaller to a higher degree of turbulence; on the other hand, to capture the injection conditions at which cavitation starts (critical cavitation conditions). Those conditions are expected to be different for each nozzle, and therefore the flow parameters describing the nature of the flow could behave in a different manner depending on the geometry.

With respect to the fluid properties introduced in the calculations, the density and viscosity values were taken from a commercial diesel fuel (Repsol CEC RF-06-99) at $25^{\circ} \mathrm{C}$. The liquid 
compressibility was calculated from speed of sound measurements in diesel fuel and the vapour properties have been obtained from a similar fuel from Kärrholm et al. in [24].

\subsection{Cavitation model validation.}

As stated in the introduction, the code has been extensively validated using experimental measurements of a standard nozzle with circular orifices. For that nozzle, mass flow measurements and momentum flux measurements were carried out. It is important to remark that momentum flux, apart from being one of the most important parameters that control the air-fuel mixing process in the spray ([25][26]), provide, in combination with mass flow measurements, important information such as effective injection velocity or effective injection section.

In Figure 6, a comparison between experiments and simulations in terms of mass flow rate, momentum flux and effective velocity is shown for a nozzle with six cylindrical orifices with a diameter of $170 \mu \mathrm{m}$.

The mass flow rate tests were performed with an Injection Rate Discharge Curve Indicator commercial System, whose measuring principle is the Bosch method. Four different backpressures were used (3, 5, 7 and $9 \mathrm{MPa})$, keeping the injection pressure constant at $160 \mathrm{MPa}$.

The averaged values of the mass flow profiles during the time in which the needle is fully open are plotted in the upper part of Figure 6 (represented with triangles) together with the numerical simulations results for the needle lift $250 \mu \mathrm{m}$ (represented with rhombus). These values have been represented as a function of the squared root of the pressure drop, being the pressure drop the difference between the injection pressure and the backpressure. The fact that the mass flow is constant for all the pressure conditions indicates that the nozzle is cavitating ([15][19][27][28]). The errors between the experimental and numerical results displayed above each couple of symbols are 
always between 2 and 5\% approximately, which means a high degree of agreement with experimental data.

Momentum flux can be measured in a test rig based on a pressurized chamber with a calibrated piezo-electric sensor. This sensor measures the force of the spray injected, which is equivalent to the spray momentum flux ([29]). The experimental results of the momentum flux tests are represented with triangles against CFD results in the bottom part of Figure 6 on the left. As can be seen, the deviation between the experimental values and momentum flux results predicted by the code at full needle lift for all the validated pressure conditions is less than $2 \%$, showing the great ability of the code to reproduce the behaviour of the flow.

Finally, dividing the momentum flux by the mass flow rate, the effective injection velocity at the nozzle outlet is obtained. The results of the effective velocity obtained experimentally against CFD results can be seen in the bottom part of Figure 6 on the right.

For all the points simulated, the code underestimates the effective velocity with a maximum deviation of $5 \%$. Taking into account the mass flow, momentum flux and effective velocity validation, the code allows to accurately predict the flow features with high level of confidence.

Details of the validation and the explanation of the experimental measurements and the experimental facilities they come from are given in [15] and [19].

\section{COMPUTATIONAL RESULTS.}

\subsection{Mass flow analysis and cavitation inception detection.}

Figure 7 shows the mass flow results for both injection pressures. Each point corresponds to a different backpressure. Although slight differences can be observed between the nozzles because of 
the wide range of variation of the mass flow with the backpressure, the Elip-H2 nozzle presents the highest mass flow for all injection conditions in general terms, whereas the Elip-V1 nozzle has the lowest mass flow. These differences will be better observed and analysed when comparing the results in terms of dimensionless parameters (values comprised between 0 and 1).

Aside from the differences found between nozzles, a similar behaviour for all nozzles can be observed for both injection pressures: the mass flow increases linearly with the square root of the pressure differential $\left(\sqrt{P_{i}-P_{b}}\right)$ until a point where it stabilizes. At this point, a flow choking occurs, which is equivalent to saying that it remains unchanged whichever the backpressure. Pressure conditions needed to reach this situation are called critical cavitation conditions (CCC). The detection of the beginning of mass flow choking is often used to experimentally detect cavitation in real nozzles ([13][15][19][30][31][32]). The critical cavitation conditions depend on the geometry ([19][30][31][32]). The critical cavitation conditions are given in Table 3: for each injection pressure, the backpressure needed to induce the nozzle to cavitate is provided. The higher the backpressure needed, the more prone the nozzle is to cavitate. Regarding the results for $30 \mathrm{MPa}$ of injection pressure, the cavitation inception for Elip-H2 nozzle takes place when the backpressure decreases until 7.21 MPa (more prone to cavitate), whereas for Elip-V1 nozzle backpressure needs to be decreased to 7.01 MPa. If nozzles are arranged in terms of tendency to cavitation we find: Elip-H2, circular, Elip-H1, Elip-V2 and Elip-V1 (with small differences between the circular and horizontal elliptical nozzles). As far as the results for the injection pressure of $160 \mathrm{MPa}$ are concerned, the same order is obtained, except for Elip-H2 and circular nozzles, which in this case, interchange their position at high Reynolds number with respect to the order established for low injection pressure conditions. Again, there are small differences between circular and horizontal elliptical nozzles.

As a first important result, it can be established that, as far as cavitation inception is concerned, 
major axis horizontally-oriented elliptical nozzles are more prone to cavitate than major axis vertically-oriented elliptical nozzles. In addition, the cavitation tendency found in the former is similar to circular nozzles.

This behaviour is related to the deflection the flow experiences when it enters the orifice through the upper corner as seen from the needle seat. In the case of major axis vertically-oriented elliptical nozzles, the entry of the flow to the orifice, which preferably takes place through its upper part, is facilitated. Thus, they are less prone to cavitate than the other cases. Considering only the major axis vertically-oriented elliptical nozzles, that proneness is higher the larger the eccentricity is (ElipV2 is more prone to cavitate than Elip-V1). In the case of the major axis horizontally-oriented elliptical nozzles, the deflection of the streamlines in the upper corner of the orifice inlet is more important than that of the vertically-oriented ones. Thus, this kind of nozzle is more prone to cavitate. In addition, as it happened with the vertically-oriented nozzles, the higher the eccentricity is, the higher the proneness to cavitate (Elip-H2 is more prone to cavitate than Elip-H1).

Consequences of this behaviour will be analyzed in the following sections.

\subsection{Comparison in terms of Momentum flux and effective velocity.}

In Figure 8, momentum flux for both injection pressures and all backpressures is depicted against the square root of pressure drop for all nozzles. As can be seen for all nozzles, momentum flux increases with the squared root of pressure drop, $\sqrt{P_{i}-P_{b}}$. However, unlike the mass flow results, momentum flux does not suffer any collapse with cavitation development [29]. As in the case of mass flow, although there are small differences between nozzles, the Elip-H2 nozzle has the highest momentum flux in general terms, whereas the Elip-V1 nozzle presents the lowest. 
With mass flow and momentum flux data, the effective injection velocity can be calculated by dividing the momentum flux by the mass flow. This effective velocity is plotted as a function of the pressure drop in Figure 9.

In the Figures it is easy to appreciate an increase in the slope of the curve when cavitating conditions are reached. For instance, in the case of $30 \mathrm{MPa}$, according to Table 3, the backpressure for reaching cavitating conditions is around 7.01-7.21 MPa depending on the nozzle geometry. Thus the value of $\sqrt{\Delta \mathrm{P}} \approx 4.8 \mathrm{MPa}$. For higher values of $\sqrt{\Delta \mathrm{P}}$, the nozzles cavitate and the change in the slope means that the increment in effective velocity is higher than it would be expected if only the increment of pressure drop was considered. This behaviour is one of the most important consequences of cavitation and is due to the viscosity reduction in the zone occupied by the vapour phase along the orifice wall, which in turn reduces the friction losses in the channel. This finding is experimentally and numerically analyzed in [15]. If the nozzles are compared in terms of effective velocity, higher injection velocities are achieved specially in cavitating conditions for nozzle ElipH2, following nozzles Elip-H1 and circular, and finally Elip-V2 and Elip-V1.

\subsection{Flow coefficients comparison.}

Flow coefficients are useful to analyze the behaviour of the flow. Flow coefficients can be represented against the Reynolds number or a cavitation number if this phenomenon is susceptible to occur, as is the case in the tested nozzles.

Figure 10 displays the discharge coefficient, velocity coefficient and area coefficient for all nozzles versus the Reynolds number. Results for the injection pressure of $30 \mathrm{MPa}$ and $160 \mathrm{MPa}$ are depicted on the left and on the right of the Figure, respectively. The Reynolds number has been calculated using equation (13), which depends on hydraulic diameter, $D_{H}$, the previously analysed effective velocity, $u_{\text {eff }}$ and the kinematic viscosity, $v$. Hydraulic diameter is evaluated using 
equation (14):

$R e=\frac{D_{H} u_{e f f}}{v}$

$D_{H}=\frac{4 A}{P}$

where the values of wetted perimeter have been calculated and are given in Table 1.

Obviously, the hydraulic diameter matches the geometrical diameter for the circular nozzle, but it has been evaluated for the different elliptical nozzles. As equation (14) reflects, for the same conditions of injection pressure and backpressure, the variation in the wetted perimeter amongst the nozzles induces variations on the hydraulic diameter up to a 6\% from Elip-V2 and Elip-H2 nozzles when compared to Elip-V1 and Elip-H1; and up to a 9\% if the former is compared to the circular one. As a consequence, these differences will be transmitted to the Reynolds number for a given condition of injection pressure and backpressure. This fact will have repercussions on the hydraulic behaviour of the nozzles, as it will be analyzed in short.

Both at low and high injection pressures, in the flow coefficients graphs (recall Figure 10) two different zones can clearly be distinguished: a zone corresponding to non-cavitating conditions (low or medium Reynolds number) and a zone corresponding to cavitating conditions (high Reynolds number).

Attending to the results, the following considerations can be made regarding the three flow coefficients, depending on whether the nozzle is in cavitating or non-cavitating conditions.

\subsubsection{Discharge coefficient}

With regard to the discharge coefficient, which is representative of the global losses in the nozzle, it shows a quite stable behaviour in the non-cavitating region, reaching maximum values of about 
0.85 depending on the nozzle. The maximum value (which implies lower global losses) corresponds to the Elip-H2 nozzle, whereas the lower value (higher level of losses) is found for the Elip-V1 nozzle. The rest of nozzles are found in between, with a similar behaviour both at low and high pressure. The major axis horizontally-oriented elliptical nozzles exhibit a higher discharge coefficient than the major axis vertically-oriented ones. The circular nozzle displays intermediate values.

In the cavitating zone, due to the mass flow collapse, the discharge coefficient experiences an abrupt drop. This drop starts at the point corresponding to the cavitation inception, characterized by a critical Reynolds number related to the injection conditions displayed in Table 3. The lowest critical Reynolds numbers at which cavitation starts are found for Elip-H2 and Elip-V2 nozzles, whereas the highest values are given for the Elip-H1 and Elip-V1 nozzles. The circular nozzle shows intermediate values, but closer to the Elip-H2 and Elip-V2 group. A lower critical Reynolds number implies that the discharge coefficient fall due to cavitation begins at lower Reynolds numbers for the corresponding nozzles. Thus, if the discharge coefficients are compared for a given Reynolds number in cavitating conditions, the lowest values are obtained for Elip-H2 and Elip-V2 nozzles and the highest ones for Elip-H1 and Elip-V1 nozzles. This means that, if they are compared for a certain Reynolds number (which implies different injection pressure and backpressure conditions) high enough to bring all the nozzles to cavitating conditions, the cavitation intensity is higher in the Elip-H2 nozzle, followed by Elip-V2, circular, Elip-V1 and finally Elip-H1.

This behaviour will have an impact on the effective area and effective velocity of injection, as it will be seen next.

\subsubsection{Area and velocity coefficients}

As seen in Figure 10, the area coefficient takes values equal to one in non-cavitating conditions 
([15][19]). Therefore, for these conditions, the velocity coefficient values equal the discharge coefficient ones. As was the case for the discharge coefficient, the area coefficient falls drastically once the cavitation phenomenon starts. The fact that the nozzles differ on the critical Reynolds number from which this phenomenon starts to occur makes that, as it happened to the discharge coefficient, for a given Reynolds number beyond the critical one, Elip-H2 and Elip-V2 nozzles show a lower area coefficient. On the other hand, Elip-H1 and Elip-V1 exhibit a higher value of this coefficient. As previously stated, this fact bears on the different intensity of the cavitation found if the nozzles are compared for a given Reynolds number.

The velocity coefficient behaviour is in agreement with the results just analyzed. Its value equals the discharge coefficient for non-cavitating conditions, since the area coefficient equals the unity. In cavitating conditions, its increase is greater the higher the cavitation intensity is. Thus, for a given Reynolds number, the highest values of the velocity coefficient are found for Elip-H2, Elip-V2 and the circular nozzles, while the lower values are found for the elliptical nozzles of a lower eccentricity Elip-H1 and Elip-V1.

The same results can be analyzed if the coefficients here studied are depicted against the cavitation number.

There are several definitions for the cavitation number ([19][30][32]). One of them is the parameter $K$, defined as a function of the injection pressure $P_{i}$, the backpressure $P_{b}$, and the vaporisation pressure $P_{\text {vap }}$,as equation (15) states. Given that the fuel vaporisation pressure is much lower than the injection pressure, it is usual to disregard the term $P_{\text {vap }}$ in the numerator.

$$
K=\frac{P_{i}-P_{v a p}}{P_{i}-P_{b}}
$$

The way this parameter is defined, as the backpressure is reduced for a given pressure, the 
denominator grows larger and the numerator remains constant. This means that, the greater the pressure difference the nozzle is submitted to, the lower the value of $K$. The value of $K$ related to the critical cavitation conditions is named as the critical cavitation number, $K_{\text {crit }}$. Attending to the critical discharge conditions depicted in Table 3, the value of $K_{\text {crit }}$ of the studied nozzles is found among 1.1 and 1.2 depending on the nozzle and the injection pressure. If the backpressure is further reduced once those conditions are reached, $K$ takes lower values than the critical one, reaching $K=$ 1 when the backpressure equals the unity, thus reaching the maximum intensity of cavitation for the injection pressure level considered.

It is typical to represent the non-dimensional flow parameters as a function of the cavitation number instead of the Reynolds number when the influence of the cavitation is treated ([28][29] [30][31][32][33][34][35][36]). The flow coefficients are plotted against the square root of $K$ in Figure 11. Even though the differences found among the nozzles and the behaviours explained for the different flow coefficients are essentially the same, a collapse of the operating points with a strong linear behaviour is noticed in the cavitating zone. The linear behaviour of the discharge coefficient with the square cavitation number $K$ in cavitating conditions was first introduced by Nurick [35] and has been contrasted in several experimental works ([28][29][30][31][34][35]). In particular, all of them observed a linear behaviour when plotting the discharge coefficient of cavitating nozzles against the cavitation number, $K$, in logarithmic scale. The slope of that line was 0.5. Although the logarithmic scale for $K$ is preferable, the same result is obtained when plotting the discharge coefficient against the square root of the cavitation number in a standard linear scale as in the results presented here.

From this new representation it is deduced that the differences between nozzles in terms of $K$, even when they exist, are less significant than those in terms of the Reynolds number. 


\subsection{Comparison in terms of cavitation morphology.}

In order to compare the morphology of the cavitation phenomenon in the different nozzles, mean images of the distribution of cavitation along the orifices of the different nozzles are displayed in Figure 12. This figure shows the zone with vapour mass fraction $(\gamma)$ between 0.1 and 1 , thus representing the zone occupied by vapour. Results correspond to the injection pressure of $30 \mathrm{MPa}$ and three different backpressures (3 MPa, $5 \mathrm{MPa}$ and $7 \mathrm{MPa}$ ). On the basis of Table 3, the last backpressure (7 MPa) would be representative of the beginning of the mass flow collapse if the differences from nozzle to nozzle were neglected. The backpressures of 5 and 3 MPa would be representative of a higher grade of cavitation intensity. Having a look at the graphs corresponding to $P_{b}=7 \mathrm{MPa}$, the morphology agrees with what was said in section 4.1 (Table 3). The intensity of cavitation is greater in the Elip-H2 nozzle, followed by the circular one, Elip-H1, Elip-V2 and ElipV1. As it was established there, the differences the circular has with respect to the major axis horizontally oriented elliptical nozzles are lower than those it has with respect to the major axis vertically oriented ones. If the attention is drawn to the lower backpressures, which result on a higher cavitation development, the differences at the morphology are reduced. However, a clear difference between the major axis horizontally oriented elliptical nozzles and the vertically oriented ones is noticed. In the former ones, the vapour zone appears closer to the wall, whereas the vapour zone is more displaced towards the centre of the nozzle for the latter. This behaviour can be observed more clearly in Figure 13, where the vapour mass fraction is depicted in the same operating conditions as the Figure 12 but having a look only at the middle section of the nozzle. The cavitation morphology for the circular nozzle is closer to the major axis horizontally oriented elliptical nozzles.

This remark is also consistent with the effective velocity of injection results. Indeed, as it has been stated previously, one of the consequences of the cavitation is the reduction of the wall friction and 
an increase in the injection effective velocity. This behaviour will be more important if the vapour zone is found along the orifice wall as it happens in the case of the major axis horizontally oriented nozzles and in the circular one, where the vapour extends along the whole upper part of the orifice. For these nozzles, the effective velocity is higher than that of the major axis vertically oriented ones for given injection conditions, as it was established in Figure 9.

It must be noted that the calculations in this work have been performed in steady-state conditions, even when the diesel injection process is, indeed, an unsteady phenomenon. However, the influence of the needle lift on the injection process was already studied by the authors in [19]. It was found that, for low needle lifts, cavitation appears in the area upstream the inlet hole section, where the needle closes against the nozzle wall. It is not until the needle reaches medium-high needle lifts that the vapour bubbles are displaced towards the nozzle orifice itself, showing a pattern similar to that depicted in Figures 12 and 13. The inclusion of the transient stage in the calculations would mainly affect the region upstream the orifice, which is the same for all the nozzles studied. Since this work intends to compare the different elliptical geometries, the transient phase is not expected to add any additional findings to the study.

\section{SYNTHESIS OF RESULTS. MIXING PROCESS ANALYSIS.}

\subsection{Synthesis of results.}

The peculiarities of the flow inside 5 nozzles (a circular section one and 4 elliptical ones) have been studied along the preceding sections. The analysis has been focused on the study of the cavitation phenomenon, its inception, morphology and its repercussion on the flow, condensing this last information in the three non-dimensional parameters. Varied injection conditions have been analyzed nevertheless in order to offer a global vision that includes both cavitating and non- 
cavitating conditions.

The following partial results have been extracted from the analysis

\subsubsection{Non-cavitating conditions.}

For certain conditions of injection within the non-cavitating regime, the major axis horizontally oriented elliptical nozzles have a higher discharge coefficient than the vertically oriented ones. In either of them, the discharge coefficient is higher the greater the eccentricity. The values for the circular nozzle are located in between both kinds of nozzles. This fact constitutes a first positive point for the major axis horizontally oriented elliptical nozzles when compared to the conventional ones, since it is significant of their lower level of losses.

\subsubsection{Cavitating conditions.}

The major axis horizontally oriented elliptical nozzles are more prone to cavitate. This proneness is higher the larger the eccentricity, even though it is not greater than the one corresponding to the standard circular nozzle. The major axis vertically oriented elliptical nozzles are the less prone to cavitate.

With regard to the morphology, the vapour zone is more attached to the wall in the major axis horizontally oriented elliptical nozzles, whereas it is more displaced towards the centre of the orifice in the vertically oriented ones. The cavitation morphology for the circular nozzles is very similar to the former ones. This morphology seems to be strongly related to what happens to one of the most important parameters from the subsequent mixture formation phenomenon: the effective velocity of injection. In fact, the nozzle with a greater effective velocity of injection is the major axis horizontally oriented one with the higher eccentricity, followed closely by the other horizontally oriented one and the circular. The vertically oriented ones show lower values of 
effective velocity of injection. As previously stated, the explanation lies on the decrease in viscosity in the near-wall zone, that leads to a more square velocity profile and hence a higher effective velocity ([15]). Obviously, this effect is more noticeable in the major axis horizontally oriented elliptical nozzles in agreement with the observed morphology.

\subsection{Mixing process prediction.}

As pointed out in the introduction, the elliptical nozzles are supposed to achieve a better atomization by the mere fact of increasing the fuel-air interaction zone when the fuel is injected to the combustion chamber. The perimeter of the orifice section supplied by Table 1 can provide a qualitative idea of the differences that could be expected from this point of view. In fact, the experimental results on the spray behaviour in this kind of nozzles point in that direction, as it has been reflected in the introduction section at the beginning of this article.

However, that improved atomization, that would imply a better mixing process, may be affected by other flow parameters that can be influenced by the presence or absence of cavitation. Indeed, the most important and influencing parameters in the mixture formation are the effective velocity of injection and the spray spreading angle ([13][28]). The higher both parameters are, the lower the mixing characteristic time and the mixing characteristic length ([13]), thus improving the mixture and combustion process.

Throughout the study, it has been seen that the cavitation provokes a substantial increase on the effective velocity of injection, and that the major axis horizontally oriented elliptical nozzle of higher eccentricity (Elip-H2) is the one that attains a higher value of this parameter. On the other hand, it is known from previous experimental studies that the cavitation produces a noticeable increase of the spray spreading angle ([28][32][37][38][39]).

Attending to the injection effective velocity values and the cavitation susceptibility and its 
consequences on the spray spreading angle, a qualitative order on the mixing process quality could be established. Taking into account all the results, in general terms, everything seems to point to a better mixing process for the major axis horizontally oriented elliptical nozzles and a worse process for the vertically oriented ones. The quality of the mixing process for the circular nozzle would take intermediate values. If it was intended to establish an order from higher to lower quality solely due to the velocity and hypothetical angle it would be find that: Elip-H2 > Circular = Elip-H1 > ElipV2 > Elip-V1. Besides, the previously commented effect of the higher fuel-air interaction of the elliptical nozzles over the circular ones would also have to be considered, since it could amplify the differences among the major axis horizontally oriented elliptical nozzles over the circular ones and reduce the differences or even swap the order of the major axis vertically oriented elliptical nozzles when compared to the circular one.

The experimental results extracted from the literature suggest a better mixing process for the elliptical nozzles when compared against the circular ones. In addition, the experimental results found in the literature for elliptical nozzles mostly refer to major axis horizontally oriented nozzles. The results obtained in this paper would therefore be in agreement with those existing in the literature.

\section{CONCLUSIONS}

The main conclusions of this study are summarized in the following points:

- The major axis horizontally oriented elliptical nozzles have a higher discharge coefficient than the vertically oriented ones. The circular nozzles adopt intermediate values.

- The proneness to cavitation is greater for the major axis horizontally oriented elliptical 
nozzles, with a similar level to the circular nozzles. The major axis vertically oriented elliptical nozzles are less prone to cavitate.

- The effective velocity of injection is higher for the major axis horizontally oriented elliptical nozzles, closely followed by the circular ones. The major axis vertically oriented elliptical nozzles are associated to the lowest values of this parameter.

- The cavitation morphology is similar for the major axis horizontally oriented elliptical nozzles and the circular ones, observing vapour structures stuck to the upper wall of the orifice. In the case of major axis vertically oriented elliptical nozzles, the cavitating structures are driven towards the central zone.

- Bearing in mind the influencing parameters in the mixing process, a better quality is presupposed for the major axis horizontally oriented nozzles whereas a worse quality is expected for the vertically oriented ones.

- The aforementioned results agree with the experimental studies found in the literature.

\section{ACKNOWLEDGMENTS}

This work was partly sponsored by “Vicerrectorado de Investigación, Desarrollo e Innovación” of the "Universitat Politècnica de Valencia" in the frame of the project "Estudio de la influencia del uso de combustibles alternativos sobre el proceso de inyección mediante GRID computing (FUELGRID)”, Reference SP20120396 and by “Ministerio de Economía y Competitividad” in the frame of the project “Comprensión de la influencia de combustibles no convencionales en el proceso de inyección y combustión tipo diesel”, reference TRA2012-36932. This support is gratefully acknowledged by the authors. 
The authors would like to thank the computer resources, technical expertise and assistance provided by the Universidad de Valencia in the use of the supercomputer “Tirant”.

\section{REFERENCES}

[1] J. B. Heywood, Internal combustion engine fundamentals, McGraw-Hill Publishing, 1988, ISBN: 007028637X

[2] A.H, Lefèbvre, Atomization and Sprays, Hemisphere, 1989

[3] H. Hiroyasu; M., Arai, Structures of fuel sprays in Diesel engines, SAE Paper 900475, 1990.

[4] D.L. Siebers, Scaling liquid-phase fuel penetration in Diesel sprays based on mixing limited vaporization, SAE Paper 1999-01-0528, 1999.

[5] Y. Wakuri, M. Fujii, T. Amitani, R. Tsuneya. Studies of the penetration of a fuel spray in a diesel engine. Bull JSME 3(9) (1960); pp:123-30.

[6] JC. Dent. A basis for the comparison of various experimental methods for studying spray penetration, SAE Paper 710571, 1971.

[7] C. Ho and E. Gutmark, Vortex induction and mass entrainment in a small-aspect-ratio elliptic jet, J. Fluid Mech., 208 (1987), pp. 382-405.

[8] F. Hussain and H.S. Hussain, Eliptic jets. Part 1, Characteristics of unexcited and excited jets”, Fluid Mech., 208 (1989), pp. 257-320.

[9] Y. Gong, L. Changwen, H. Yezhou, P. Zhijun, An experimental study on droplet size characteristics and air entrainment of elliptic sprays, SAE Paper 982546. 
[10] C.-W. Lee, I. Kim, K.-W. Koo, J. Park and Y. Lee, Experimental study of the effects of nozzle hole geometry for a DI diesel engine, ICLASS, 2006.

[11] J. G. Hong, W. Kun, S. R. Kim, C.W. Lee, Effect of cavitation in circular nozzle and elliptical nozzles on the spray characteristic, Atomization and sprays, 20(10) (2010): pp. 887-886.

[12] A. Matsson, L. Jacobssonand S. Andersson, The effect of elliptical nozzle holes on combustion and emission formation in a heavy duty diesel engine, SAE Paper, 2000-011251.

[13] R. Payri., F.J. Salvador, J. Gimeno, L.D. Zapata, Diesel nozzle geometry influence on spray liquid-phase fuel penetration in evaporative conditions, Fuel,87 (2007), pp. 1165-1176.

[14] OpenFOAM is a r ${ }^{\circledR}$ registered trade mark of OpenCFD Limitedhttp://www.opencfd.co.uk/openfoam/index.html

[15] F. Payri, R. Payri, F.J. Salvador, J. Martínez-López, A contribution to the understanding of cavitation effects in diesel injector nozzles through a combined experimental and computational investigation, Computer and Fluids, 58 (2012), pp. 88-101.

[16] T.J. Chung, Computational Fluid Dynamics, second edition, Cambridge University Press (2010) ISBN 978-0-521-76969-3.

[17] H.B. Stewart, B. Wendroff, Two-phase flow: models and methods, J. Comput. Phys., vol. 56 (1984), pp. 363-409.

[18] V. Yakhot, S.A. Orszag, S. Thangam, T.B. Gatski and C.G. Speziale, Development of turbulence models for shear flows by a double expansion technique, Phys. Fluids A 4 (1992), 1510. 
[19] F.J. Salvador, J. Martínez-López, M. Caballer, C. de Alfonso, Study of the influence of needle lift on the internal flow and cavitation phenomenon in diesel injector nozzles by CFD using RANS methods, Energy conversion and management, vol. 66 (2013), pp. 246-256.

[20] V. Macián, R. Payri, X. Margot, F.J. Salvador, A CFD analysis of the influence of diesel nozzle geometry on the inception of cavitation, Atomization and sprays, vol. 13 (2003), pp. 579-604.

[21] Spanish National Grid Initiative http://www.es-ngi.es/

[22] European Grid Initiative http://www.egi.eu

[23] Tirant Node http://www.bsc.es/annualreport/2009/wikiar.bsc.es/index.php5/Universidad_de_Valencia_-_Tirant_Node.html

[24]F. Peng Kärrholm, H. Weller and N. Nordin, Modelling injector flow including cavitation effects for Diesel Applications, Proceedings of FEDSM2007, 5th Joint ASME/JSME Fluids Engineering Conference, July 30- August 2, San Diego, California, USA.

[25] J.M. Desantes, R. Payri, J.M. García, F.J. Salvador, A contribution to the understanding of isothermal diesel spray dynamics, Fuel, 86 (2007), pp. 1093-110.

[26] J.M. Desantes, F.J. Salvador, J.J. López, J. de la Morena, Study of mass and momentum transfer in diesel sprays based on a theoretical derivation, Experiments in fluids, vol. 50 (2) (2011), pp. 236-246.

[27] F.J. Salvador, S. Hoyas, R. Novella, J. Martínez-López, Numerical simulation and extended validation of two-phase compressible flow in diesel injector nozzles, Proceedings of the institution of mechanical engineers part-D-Journal of automobile engineering, vol. 225, (2011), pp. 545-563. 
[28] F.Payri, V. Bermúdez, R. Payri, F.J. Salvador, The influence of cavitation on the internal flow and the spray characteristics in diesel injection nozzles, Fuel, 83 (2004), pp. 419-431.

[29] R. Payri, J.M. García, F.J. Salvador, J. Gimeno, Using spray momentum flux measurements to understand the influence of diesel nozzle geometry on spray characteristics, Fuel, vol 84 (2005), pp- 551-561.

[30] C. Soteriou, R. Andrews, M. Smith, Further studies of cavitation and atomization in diesel injection. SAE Paper 1999-01-1486.

[31] D.P. Schmidt, C.J. Rutland, M.L. Corradini, A fully comprenssible two-dimensional model of high speed cavitating nozzles, Atomization and sprays, 9 (2009).

[32] A. Sou, S. Hosokawa, A. Tomiyama, Effects of cavitation in a nozzle on liquid jet atomization. International Journal of heat of mass transfer, 50 (2007), pp. 3575-3582.

[33] V. Bermúdez, R. Payri, F.J. Salvador, Study of the influence of nozzle seat type on injection rate and spray behaviour, Proceedings of the institution of mechanical engineers Part-D, Journal of automobile engineering, 219 issue D5 (2005), pp. 677-689.

[34] D.P. Schmidt and M.L. Corradini, The internal Flow of Diesel Fuel Injector Nozzles: a Review, International Journal of Engine Research, 2 (1) (2001).

[35] W.H. Nurick, Orifice cavitation and its effect on Spray Mixing, Journal of Fluids Engineering 98 (1976).

[36]C. Arcoumanis, H. Flora, M. Gavaises, N. Kampanis and R. Horrocks, Investigation of cavitation in a vertical multi-hole diesel injector. SAE Paper 1999-01-0524. 
[37] J.M. Desantes, R. Payri, F.J. Salvador and J. De la Morena, Influence of cavitation phenomenon on primary break-up and spray behavior at stationary conditions, Fuel, 89 (2010), pp. 3033-3041.

[38] R. Payri, F.J. Salvador, J. Gimeno, J. de la Morena, Effects of nozzle geometry on direct injection diesel engine combustion process, Applied thermal engineering, 29 (2009), pp. 2051-2060

[39] S. Som, S.K. Aggarwal, E.M. El-Hannouny and D.E. Longman. Investigation of nozzle flow and cavitation characteristics in a diesel injector, Journal of Engineering for Gas and Turbines and Power, 132 (2010). 
Table 1: Nozzle's geometrical characteristics.

Table 2: Inlet-Outlet pressure boundary conditions.

Table 3: Critical cavitation condition inception

Figure 1: Nozzle's geometrical parameters

Figure 2: Nozzle's geometry

Figure 3: Boundary conditions for numerical simulations.

Figure 4: Detail of the mesh used in simulations

Figure 5: Mean y + values within the whole computational domain for all simulated nozzles

Figure 6: Cavitation model validation for a cilindrical nozzle

Figure 7: Mass Flow for the injection pressures of 30 and $160 \mathrm{MPa}$

Figure 8: Momentum flux for the injection pressures of 30 and $160 \mathrm{MPa}$

Figure 9: Effective velocity for the injection pressures of 30 and $160 \mathrm{MPa}$

Figure 10: Flow coefficients against Re number

Figure 11: Flow coefficients against the square root of cavitation number

Figure 12: Vapour mass fraction contour for the injection pressure of $30 \mathrm{MPa}$

Figure 13: Vapour mass fraction at the middle section of the nozzle for the injection pressure of 30 $\mathrm{MPa}$ 


\begin{tabular}{|c|c|c|c|c|c|c|c|c|c|}
\hline 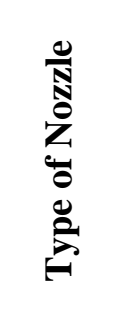 & 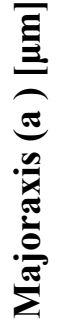 & 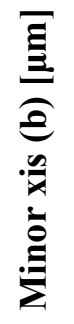 & 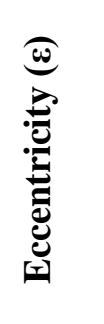 & 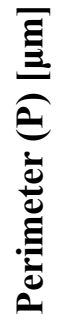 & 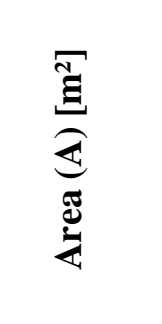 & 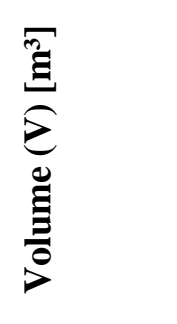 & 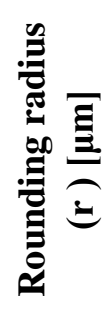 & 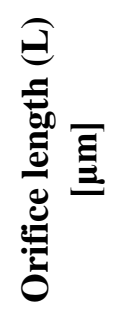 & 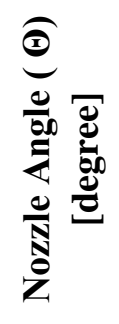 \\
\hline Circular & 85 & 85 & 0 & 534 & $2.270 \mathrm{e}-8$ & $2.270 \mathrm{e}-11$ & 30 & 1 & $72.5^{\circ}$ \\
\hline Elip-H1 & 103 & 70 & 0.734 & 548 & $2.265 e-8$ & 2.265e-11 & 30 & 1 & $72.5^{\circ}$ \\
\hline Elip-V1 & 103 & 70 & 0.734 & 548 & $2.265 e-8$ & 2.265e-11 & 30 & 1 & $72.5^{\circ}$ \\
\hline Elip-H2 & 120 & 60 & 0.866 & 581 & $2.262 \mathrm{e}-8$ & $2.262 \mathrm{e}-11$ & 30 & 1 & $72.5^{\circ}$ \\
\hline Elip-V2 & 120 & 60 & 0.866 & 581 & $2.262 \mathrm{e}-8$ & $2.262 \mathrm{e}-11$ & 30 & 1 & $72.5^{\circ}$ \\
\hline
\end{tabular}

Table 1: Nozzle's geometrical characteristics.

\begin{tabular}{|l|l|}
\hline Injection Pressure [MPa] & Backpressure [MPa] \\
\hline 30 & $3-5-7-9-10-15-20$ \\
\hline 160 & $3-5-7-9-10-20-30-40-50-60-70-80-90-100-110-120-130$ \\
\hline
\end{tabular}

Table 2: Inlet-Outlet pressure boundary conditions.

\begin{tabular}{|c|c|c|}
\hline & $30 \mathrm{MPa}$ & $160 \mathrm{MPa}$ \\
\hline CIRCULAR & 7.19 & 39.83 \\
\hline ELIP-H1 & 7.18 & 37.38 \\
\hline ELIP-V1 & 7.01 & 36.24 \\
\hline ELIP-H2 & 7.21 & 37.97 \\
\hline ELIP-V2 & 7.13 & 36.43 \\
\hline
\end{tabular}

Table 3: Critical cavitation condition. 